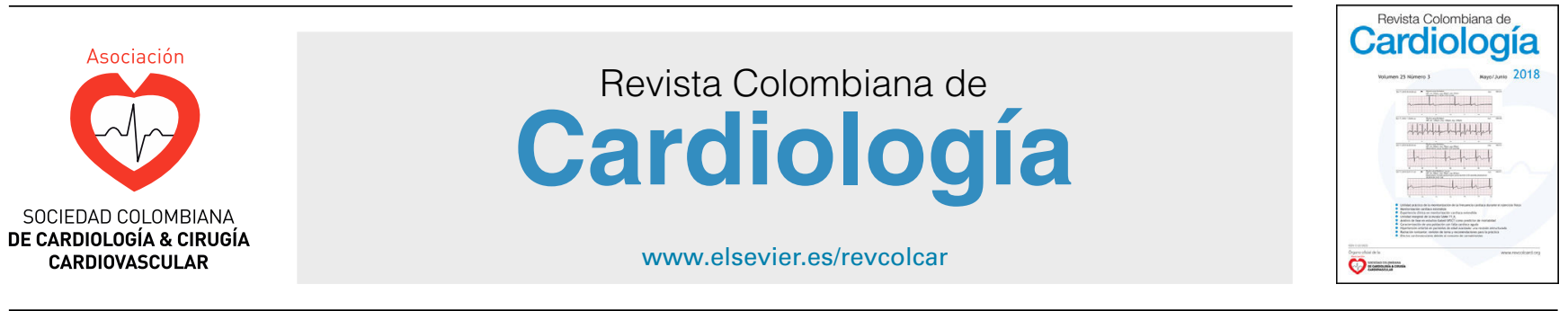

CARDIOLOGÍA DEL ADULTO - REVISIÓN DE TEMAS

\title{
Accesos vasculares femoral y radial en intervenciones coronarias percutáneas en síndrome coronario agudo y enfermedad crónica inestabilizada
}

\author{
Andrés Ramírez Vélez ${ }^{\mathrm{a}, *}$, Simón Gaviria Valencia ${ }^{\mathrm{a}}$, Nicolás Jaramillo Gómez ${ }^{\mathrm{b}}$, \\ Heidy Contreras Martínez ${ }^{\mathrm{C}}$ y Jonathan Cardona Vélez ${ }^{\mathrm{d}}$
}

\author{
a Universidad CES, Medellín, Colombia \\ b Cardiología intervencionista, Clínica Las Américas, Medellín, Colombia \\ c Epidemiología, Universidad CES, Medellín, Colombia \\ d Universidad Pontificia Bolivariana, Medellín, Colombia
}

Recibido el 15 de junio de 2017; aceptado el 2 de enero de 2018

Disponible en Internet el 28 de mayo de 2018

\section{PALABRAS CLAVE}

Accesos vasculares; Síndrome coronario agudo; Intervención coronaria percutánea

\section{KEYWORDS}

Vascular access;

Acute coronary

syndrome;

Percutaneous

coronary intervention

\begin{abstract}
Resumen La enfermedad cardiaca isquémica continúa siendo en la actualidad la principal causa de muerte en el mundo, pues genera más de 17.7 millones de decesos al año; específicamente, constituye alrededor del $30 \%$ de todas las causas de muerte en Colombia. Se han establecido diferentes estrategias de manejo que con el paso del tiempo han mostrado impacto en la disminución de la morbilidad y mortalidad por esta causa. La intervención coronaria percutánea es la que más se ha impuesto, y es en la actualidad la piedra angular para el tratamiento del síndrome coronario con o sin elevación del segmento ST. Existen diferentes sitios de acceso vascular; entre ellos los más destacados son la vía femoral y la radial. En este artículo se revisa la evidencia actual con base en estudios prospectivos y meta-análisis sobre las complicaciones y el posible compromiso de la funcionalidad de las extremidades irrigadas por los diferentes vasos de acceso.

(c) 2018 Sociedad Colombiana de Cardiología y Cirugía Cardiovascular. Publicado por Elsevier España, S.L.U. Este es un artículo Open Access bajo la licencia CC BY-NC-ND (http:// creativecommons.org/licenses/by-nc-nd/4.0/).
\end{abstract}

Radial and femoral vascular access in percutaneous coronary interventions in acute coronary syndrome and unstable chronic disease

Abstract Ischaemic heart disease still continues to be the main cause of death worldwide. It causes 17.7 million deaths, and in particular it constitutes around $30 \%$ of all-cause mortality in Colombia. Different management strategies have been established that, over time, have shown

\footnotetext{
* Autor para correspondencia.

Correos electrónicos: aramirez94@hotmail.com, aramirez9423@gmail.com (A. Ramírez Vélez).
} 
an impact in the reduction in the morbidity and mortality by this cause. Percutaneous coronary intervention has been the most effective, and is currently the cornerstone for the treatment of acute coronary syndrome with or without ST segment elevation. There are different vascular accesses, with the main ones being the femoral or radial route. In this article, the current evidence is reviewed, based on prospective studies and meta-analyses on the complications and possible compromise of functionality of the limbs irrigated by the different access vessels. (C) 2018 Sociedad Colombiana de Cardiología y Cirugía Cardiovascular. Published by Elsevier España, S.L.U. This is an open access article under the CC BY-NC-ND license (http:// creativecommons.org/licenses/by-nc-nd/4.0/).

\section{Introducción}

La enfermedad cardiaca isquémica es hoy la principal causa de muerte en el mundo según la Organización Mundial de la Salud (OMS). Se registraron alrededor de 17.7 millones de muertes en 2016 superando al cáncer, a las enfermedades respiratorias y a la diabetes con $8.8,3.9$ y 1.6 millones de muertes respectivamente. La muerte por enfermedad cardiovascular representa el $31 \%$ de todas las muertes ${ }^{1}$. Según cifras del Ministerio de Salud, en el análisis de situación en salud en Colombia del 2013 al 2015, las principales causas de muerte fueron las enfermedades del sistema cardiovascular, con incidencias de 146.16 por 100.000 habitantes, lo que representa el $29,9 \%$ del total de muertes, casi el doble de las enfermedades neoplásicas, que representaron el $17,79 \%{ }^{1}$.

Para esta condición existen tres estrategias de manejo: terapia farmacológica, intervención coronaria percutánea (ICP) y cirugía. En la actualidad la ICP es la piedra angular para el tratamiento invasivo de los pacientes con síndrome coronario agudo tanto con elevación como sin elevación del segmento ST (SCAST y SCASST) ${ }^{2,3}$; ésta ha evolucionado en gran medida en los últimos 20 años debido a la aparición de nuevas técnicas, dispositivos, medicamentos antitrombóticos y anticoagulantes, mejorando sus tasas de éxito y disminuyendo la incidencia de complicaciones isquémicas y hemorrágicas asociadas a su uso ${ }^{4}$.

Hoy día se utilizan varios sitios de acceso vascular, entre los que se destacan las vías femoral, braquial y radial ${ }^{4}$. La vía femoral es un acceso que goza de gran aceptación mundial, debido a su trayectoria histórica y amplio conocimiento en el manejo de sus complicaciones ${ }^{5-8}$. Sin embargo, desde la última década el acceso radial ha recobrado su uso debido a su menor tasa de complicaciones fatales comparada con el acceso femoral ${ }^{4}$. En los estudios de seguimiento de complicaciones vasculares en la intervención de la enfermedad coronaria, uno de los mayores eventos adversos es la presencia de sangrados, los cuales van de la mano con la incidencia en mortalidad y están directamente asociados al sitio de acceso escogido? .

La ICP como cualquier terapia invasiva está sujeta a complicaciones dependientes de la experiencia del clínico, el sitio de acceso, las características del paciente, la duración y el tipo de procedimiento. Durante los últimos veinte años se han publicado múltiples estudios como RIVAL, RIFLE STEACS y MATRIX, entre otros, los cuales han comparado la efectividad y la tasa de complicaciones de las diferentes técnicas de acceso y han concluido que a pesar de la aparición de nuevos avances en terapias antitrombóticas, anticoagulantes y la propia técnica de intervención, el sangrado sigue siendo una fuente significativa de morbilidad y mortalidad en pacientes que van a ICP por vía femoral y que por el contrario, el acceso vía radial ha mostrado un impacto positivo en el riesgo de sangrado $8,10,11$.

\section{Accesos percutáneos}

Son múltiples las alternativas para el acceso vascular; no obstante, las clásicas son el acceso femoral y radial, cada una con sus ventajas y desventajas. La elección de una u otra vía dependerá de las características del paciente, el tipo de procedimiento a realizar y las habilidades y experiencia clínica del intervencionista ${ }^{12}$.

La evidencia científica apoya cada vez más el uso del acceso radial en la ICP para el manejo de la enfermedad coronaria; sin embargo, la vía femoral fue la primera en ser descrita y por tanto cuenta con gran experiencia clínica y soporte científico, a diferencia del acceso radial, el que a pesar de los diferentes estudios que avalan su uso, carece de la misma evidencia ${ }^{4}$.

El sangrado en pacientes que son sometidos a ICP se asocia con mayor mortalidad a largo plazo, presentándose frecuentemente y de forma más significativa en aquellos que son intervenidos por vía femoral, con un incremento de 2 a 8 veces de cualquier tipo de sangrado relacionado con el procedimiento. La supervivencia en las poblaciones intervenidas disminuye semanas o meses después de iniciado el sangrado $^{13}$.

El estudio RIVAL comparó los desenlaces ocurridos entre dos sitios diferentes de acceso, fuera femoral o radial, en pacientes con síndrome coronario agudo (SCA). El desenlace primario fue el compuesto de muerte, infarto agudo de miocardio (IAM), accidente cerebrovascular o sangrado mayor no asociado a cirugía de bypass coronario a los 30 días de la intervención. Los resultados demostraron que el acceso radial se asoció con una reducción en la incidencia de hematomas grandes (HR 0,40 IC 95\%, 0,28-0,57; $p<0,0001$ ) y pseudoaneurismas con necesidad de cierre (HR 0,30 IC $95 \%, 0,13-0,71 ; p=0,006)$, comparado con el acceso vascular femoral. Concluye que ambos accesos son igual de seguros y efectivos, pero podría ser preferible el acceso radial dada su menor incidencia de complicaciones vasculares locales ${ }^{8}$. 
En el estudio RIFLE-STEACS se evaluaron los resultados finales de la comparación entre los accesos vasculares (radial vs. femoral) en 1.001 pacientes con SCAST. El desenlace primario fue el compuesto de muerte cardiaca, accidente cerebrovascular, infarto agudo de miocardio, lesión diana de revascularización y sangrado durante los primeros 30 días luego de la ICP. Los resultados demostraron que el acceso radial se asoció a una disminución significativa en la mortalidad cardiaca $(5,2 \%$ vs. $9,2 \%, p=0,020)$ y sangrado $(7,8 \%$ vs. $12,2 \%, p=0,026)$, además de una estancia hospitalaria más corta, en comparación con el acceso femoral $(p=0,03)$, concluyéndose que en pacientes con SCAST la vía radial presenta menor morbilidad y mortalidad cardiaca $^{11}$.

Otros estudios como ACCESS y MATRIX demostraron que el acceso radial es igual de seguro y factible al acceso femoral pero se asocia con mejores beneficios clínicos en términos de menor morbilidad y mortalidad cardiaca. MATRIX comparó los desenlaces del acceso radial respecto al acceso femoral en 8.404 pacientes con SCA, demostrando que los pacientes intervenidos por vía radial tuvieron menor incidencia de eventos clínicos adversos netos $(9,8 \%)$ comparados con aquellos cuya intervención se hizo vía femoral $(11,7 \%)$ (RR 0,83 IC 95\%, 0,73-0,96; $p=0,0092$ ). Esta diferencia se produjo a expensas de una reducción del sangrado mayor no relacionado a cirugía de bypass coronario, clasificado según la escala BARC (Bleeding Academic Research Consortium) (1,6\% vs. $2,3 \%$, RR 0,67 IC 95\%, 0,49-0,92; $p=0,013)$ y de todas las causas de mortalidad ( $1,6 \%$ vs. $2,2 \%$, RR 0,72 IC $95 \%, 0,53-$ $0,99 ; p=0,045)$. Concluyeron que en los pacientes con SCA que requieren manejo invasivo, el acceso radial comparado con el femoral reduce los eventos clínicos adversos netos a través de una diminución del sangrado mayor y de todas las causas de muerte ${ }^{10,14}$.

En el acceso radial, como se ha venido demostrando en los diferentes estudios, tanto las complicaciones vasculares como el sangrado se reducen de manera importante; sin embargo, este tipo de vía presenta una tendencia a tener que cambiar de estrategia en el acceso (por dificultades como arterias radiales pequeñas, tortuosas y presencia de espasmo inducido) que impacta en el éxito. Aunque las tendencias en estos últimos diez años muestran al acceso femoral como la vía preferida por los operadores, el acceso radial sigue demostrando ventajas significativas en cuanto a reducción de complicaciones vasculares y a su vez la estancia hospitalaria. Los eventos adversos tanto cardiovasculares como sangrados mayores se han logrado disminuir en los centros que realizan altos volúmenes de acceso radial ${ }^{5,11}$.

Persisten tendencias de acuerdo con sitios de trabajo y países en los que el acceso femoral es el principal sitio para punción, dada la inercia para el cambio. En Estados Unidos por ejemplo se mantiene en un 70\%, a diferencia de la mayoría de países de la unión Europea y Latino América, en donde la tendencia del acceso radial ha penetrado hasta un $80 \%{ }^{15}$. Sin embargo, la tendencia sugiere que el interés y la adopción de esta técnica en Estados Unidos está creciendo de manera apresurada más allá que la previamente observada $^{16}$.

La terapia de antiagregación plaquetaria ha venido mostrando mayor eficiencia con nuevas moléculas de potencia antiagregante superior, al mismo tiempo evaluándose regímenes combinados (antiagregación dual) que han llevado a modificar las guías de las sociedades americanas y europeas de acuerdo con las evidencias, que sugieren el uso de ácido acetil salicílico (ASA) e inhibidores P2Y12 en pacientes con SCA. Esta potencia dual asociando ASA con inhibidores P2Y12, a pesar de que contribuye a un mayor sangrado en los sitios de punción, impacta de forma positiva cuando se compara con los sangrados ocasionados al asociar anticoagulantes más inhibidores de la glicoproteína IIB-IIIA, usados anteriormente en búsqueda de potencia ${ }^{17,18}$.

De acuerdo con un metaanálisis publicado en The American Journal of Cardiology en 2016 donde se comparó la seguridad y efectividad del acceso radial vs. el acceso femoral en pacientes con SCASST, no hubo diferencias significativas en la incidencia a corto plazo de mortalidad entre accesos (OR 0,78 IC 95\%, 0,57-1,07, $p=0,12$ ). Aunque en cuanto al sangrado mayor, el acceso radial presentó reducción significativa (OR 0,52 IC 95\%, 0,36-0,73, $p=0,0002$ ), al igual que el sangrado en el sitio de acceso (OR 0,41 IC $95 \%, 0,22-0,78, p=0,007)$ y la necesidad de transfusión sanguínea (OR 0,61 IC 95\%, 0,41-0,91, $p=0,02$ ). Respecto a la mortalidad a un año fue significativamente menor en el grupo del acceso radial (OR 0,72 IC 95\%, 0,55-0,95, $p=0,02$ ). Concluye el estudio que en pacientes con SCASST el acceso radial fue asociado con menor sangrado y menos complicaciones en el sitio del acceso ${ }^{19}$.

Un reciente metaanálisis publicado en 2016 comparó los accesos vasculares femoral y radial para intervenciones coronarias en todo el espectro de enfermedad coronaria aguda. Incluyó 24 estudios para una muestra total de 22.843 pacientes y demostró que comparado con el acceso femoral, el acceso radial se asoció a disminución significativa en el riesgo de todas las causas de mortalidad (OR 0,71 IC 95\%, 0,59-0,87, $p=0,001)$, eventos adversos cardiovasculares mayores (MACE) (OR 0,84 IC 95\%, 0,75-0,94, $p=0,002$ ), sangrado mayor (OR 0,53 IC 95\%, 0,42-0,65, $p<0,001$ ) y complicaciones vasculares mayores (OR 0,23 IC 95\%, 0,16-0,35, $p<0,001)$. En conclusión, comparando el acceso femoral con el acceso radial, este último reduce la mortalidad y los MACE, y mejora la seguridad, pues disminuye el sangrado mayor y las complicaciones vasculares en todo el espectro de la enfermedad coronaria ${ }^{20}$.

\section{Complicaciones específicas del acceso femoral}

La superioridad del acceso radial sobre el femoral en los SCA ha sido avalada por varios estudios ${ }^{12}$. Sin embargo, el acceso femoral mantiene unas indicaciones claras que se imponen a la hora de escoger la vía, como son la necesidad de usar balón de contra-pulsación intra-aórtica o cuando el arco palmar no es adecuado (maniobra de Allen positiva), presencia de tortuosidad severa, ausencia de pulso o trabajo fino con la extremidad superior ${ }^{21}$.

La principal complicación del acceso femoral es el sangrado mayor, definido como aquel que requiere transfusión, soporte inotrópico, cirugía inmediata y/o la aparición de hematomas asociados a compromiso hemodinámico, siendo así un factor independiente de riesgo de muerte, transfusiones y requerimiento de unidad de cuidados intensivos (UCI) o unidad de cuidados especiales (UCE) ${ }^{12}$. En el estudio de Gómez et al., se identificaron como factores pronósticos para el desarrollo de sangrado el uso de warfarina (OR 4,7 IC 
Tabla 1 Principales factores asociados a sangrado post ICP

1. Mayores de 70 años
2. Género femenino
3. Enfermedad renal crónica
4. Anemia
5. Accidente cerebrovascular previo
6. Bajo peso corporal
7. Diabetes
8. Infarto agudo de miocardio con cambios en el segmento
ST
9. Hipertensión arterial crónica
10. Intervención urgente
11. Procedimientos invasivos (balón de contrapulsación
intraaórtica)
12. Enfermedad coronaria multivaso
13. INR $>2,5$

Adaptada de: Gómez R, et al. Factores asociados a la presencia de sangrado clasificados por el Bleeding Academic Research Consortium en pacientes con síndrome coronario agudo sometidos a intervención coronaria percutánea

95\%, 1,35-16,83), sexo femenino (OR 2, 15 IC 95\%, 1, 18-3,93) y el uso de tirofibán (OR 2,59 IC 95\%, 1,4- 4,08) (tabla 1) ${ }^{22}$. La utilización cada vez mayor de introductores vasculares de menor calibre ha disminuido la tasa de sangrados mayores $y$ reparos vasculares en la intervención por vía femoral ${ }^{23}$.

Otras de las complicaciones del acceso femoral son: hemorragia retroperitoneal, pseudoaneurismas, fístulas arteriovenosas, oclusión arterial, neuropatía femoral e infección. Algunos eventos en particular pueden estar asociados a la técnica como punción debajo de la bifurcación generando pseudoaneurismas, hematomas y fístulas arteriovenosas, mientras que la hemorragia retroperitoneal es causada por punciones más altas en la arteria femoral ${ }^{24}$.

\section{Complicaciones específicas del acceso radial}

Dentro de los eventos adversos más comunes en el acceso radial está la oclusión de la arteria radial, que pocas veces presenta signos clínicos debido a la existencia del arco palmar, donde la arteria cubital suple de forma eficiente la circulación de la arteria radial pero limita su uso para una futura intervención por esta vía ${ }^{25}$. Un metaanálisis realizado por Rashid et al., identificó algunos factores que aumentan el riesgo de oclusión de esta $\operatorname{arteria}^{26}$ (tabla 2 ).

Como se expresó con anterioridad, la oclusión arterial es la principal complicación del acceso vascular por vía radial, que oscila entre un $5 \%$ a $8 \%$, y puede llevar en algunos casos a isquemia de la mano. Otros factores asociados que facilitan su presentación son: estado de hipoperfusión prolongada asociado a hipotensión, altas dosis de vasopresores, duración prolongada de la canulación, número de intentos en la punción, introductores arteriales de grandes calibres (mayor de 5 French), enfermedad arterial periférica, jóvenes y sexo femenino. Algunos de los síntomas descritos por la oclusión arterial son disminución de la fuerza muscular, parálisis, rigidez y frialdad de extremidad distal ${ }^{27}$.

Un estudio clínico aleatorizado que incluyó 171 pacientes evaluó los desenlaces clínicos tras el uso de catéteres 5 vs. $6 \mathrm{Fr}$ en ICP vía radial. El desenlace primario fue el
Tabla 2 Factores asociados a oclusión de la arteria radial

1. Arteria de calibre pequeño

2. Uso de introductores vasculares de grandes

3. Género femenino

4. No uso de heparina

5. Diabetes

6.Compresión prolongada debida a sangrado

7. Hipoperfusión prolongada asociada a hipotensión

8. Altas dosis de vasopresores

9. Duración prolongada de la canulación

10. Número de intentos en la punción

11. Introductores arteriales de gran calibre (mayores de

$5 \mathrm{Fr}$ )

12. Enfermedad arterial periférica

Adaptada de Rashid M, et al. Radial artery occlusion after transradial interventions: a systematic review and meta-analysis y assessment of collateral circulation to the hand prior to radial artery harvest.

éxito del procedimiento, logrado en un $95,4 \%$ y en un $92,9 \%$ de los pacientes con el uso de catéteres 5 y 6 Fr respectivamente. Dentro de los desenlaces secundarios, se reportaron hematomas menores sin necesidad de reparo quirúrgico o transfusiones de sangre en 1,1\% (5 Fr) y 4,8\% (6 Fr; $p=0,07)$. Se presentó pérdida del pulso radial por oclusión en el $1,1 \%$ (5 Fr) y 5,9\% (6 Fr) con una $p=0,05$. Este estudio demostró una tendencia de mayores tasas de éxito y menores complicaciones vasculares asociadas al uso de catéteres $5 \mathrm{Fr}$ vs. $6 \mathrm{Fr}$ por vía radial en pacientes sometidos a ICP ${ }^{28}$.

Otras complicaciones de la intervención por acceso radial son las hemorrágicas, hematomas, pseudoaneurismas, fístulas arterio-venosas, síndrome compartimental, disección de la arteria y ruptura vascular ${ }^{29}$.

Existen complicaciones desencadenadas por el compromiso vascular en los accesos radiales que pueden pasar inadvertidas en su inicio; estas son pérdida de la fuerza muscular y funcionalidad, detectada por pérdida de la fuerza prensil ${ }^{30}$. Pocos estudios le han dado el verdadero valor a este efecto adverso, el cual al no ser cuantificado con un dinamómetro, solo deja la idea subjetiva en los grupos de trabajo y en el paciente mismo; por tanto, no existen estadísticas que validen el verdadero impacto de esta complicación, que puede hacerse evidente e incapacitante de acuerdo con el tipo de labor que desempeñe el paciente.

Entre los pocos estudios que cuantifican este efecto adverso está el ARCUS, que evaluó tanto la pérdida de la fuerza muscular como de la funcionalidad en la mano caso en el que se realizó la intervención radial buscando proveer una visión completa en cuanto a la morbilidad desencadenada por este tipo de acceso, midiendo la funcionalidad de la extremidad superior después de la intervención en términos de fuerza, arcos de movimiento, sensibilidad, dolor, integridad anatómica y coordinación ${ }^{30}$.

El estudio incluyó una muestra de 500 pacientes, todos intervenidos por acceso radial por operadores de alta experiencia y evaluó la disfunción en la extremidad superior correspondiente al acceso. El seguimiento se hizo a las 24 horas, dos semanas, un mes y seis meses e incluyó un registro eco doppler con el fin de evidenciar la permeabilidad en la arteria radial en cada una de las evaluaciones; 
igualmente se determinaron eventos adversos como sangrado o eventos cardiacos mayores (MACE). El desenlace primario fue disfunción de la extremidad superior definida como un puntaje mayor en el Boston Carpal Tunnel Syndrome Questionnaire (BCTQscore). Los resultados aún no han sido publicados y se espera que en el trascurso del año 2017 se conozcan los datos completos, con los que se generará nuevo conocimiento sobre estos efectos adversos poco documentados o no tenidos en cuenta por los grupos de intervención en su práctica diaria ${ }^{30}$.

En una revisión sistemática en la que se analizaron 176 artículos, se describió la incidencia de complicaciones desencadenadas por el acceso radial de la extremidad superior intervenida, encontrando una incidencia alrededor del $9,6 \%$ de complicaciones generales y hasta $1,7 \%$ en la disfunción de dicha extremidad ${ }^{31}$.

En cuanto a posibles complicaciones neurológicas del acceso radial, en un metaanálisis publicado recientemente se evaluó la influencia del sitio de acceso para ICP en pacientes con SCA y sus implicaciones neurológicas y se concluyó que la intervención a través del acceso en mención no se asocia con aumento en los eventos neurológicos (RR 0,71 IC $95 \%, 0,52-0,98)^{32}$.

Durante el congreso Europeo de Cardiología de 2016 se expuso sobre las disfunciones de la extremidad superior que pasan inadvertidas en los pacientes que fueron intervenidos por vía radial. De estos datos se desprende que un $75 \%$ de los pacientes presentaron alguna manifestación clínica en la extremidad superior intervenida, y que la pérdida de la fuerza muscular cuantificada con dinamómetro, fue la principal. También se presentaron alteraciones significativas en los flujos detectados por eco doppler hasta en un 7,3\% de éstos ${ }^{33}$.

Una las recomendaciones fundamentales que surgen de estas observaciones realizadas en una población determinada, es evitar el uso de accesos radiales en pacientes que por motivos de su profesión dependan de un estado óptimo de sus extremidades superiores, como son artistas, deportistas, manejo de muletas, etc. ${ }^{33}$. Existe un gran déficit de información en este aspecto y al mismo tiempo hay poca conciencia en los grupos de intervención de estos aspectos sutiles y que pasan inadvertidos.

Por otro lado, una de las complicaciones asociadas al uso del acceso radial es la mayor exposición a radiación para el operador y el paciente. El estudio MATRIX al comparar el acceso femoral y radial, encontró que debido al uso de introductores y catéteres de menor calibre, el acceso radial se asociaba a mayor incidencia de espasmo vascular y mayor duración del procedimiento sobre todo en operadores poco experimentados, lo cual llevaba a mayor radiación al operador y al paciente y generaba una curva de aprendizaje más prolongada $^{14}$.

\section{Enfermedad renal aguda}

Con el aumento en la incidencia del uso del acceso radial para disminuir las tasas de sangrado, se esperaba que aumentara la incidencia de enfermedad renal aguda.

De esta tendencia hacia el uso de los accesos radiales, aunque se tengan algunas reacciones adversas inherentes al procedimiento, se le ha venido atribuyendo una incidencia menor de enfermedad renal aguda. La razón no es aun clara; parece que el acceso radial por sí mismo desencadena menor reacción sistémica, respecto al femoral.

El estudio AKI MATRIX, un subestudio del MATRIX, comparó el desarrollo de enfermedad renal aguda de acuerdo con el acceso vascular. El desenlace primario fue la aparición de enfermedad renal aguda, definida como aumento absoluto $(>0,5 \mathrm{mg} / \mathrm{dl})$ o relativo $(>25 \%)$ de la creatinina sérica. Se halló que la enfermedad renal aguda fue 3 veces menos prevalente cuando se usó el acceso radial (OR 0,85 IC 95\%, 0,70-1,03; $p=0,090)$. Se requirió diálisis post ICP en 6 pacientes $(0,15 \%)$ con abordaje radial y en $14(0,34 \%)$ con abordaje femoral $(p=0,0814)$, por lo que se concluyó que el acceso radial se asocia con un menor riesgo de desarrollar enfermedad renal aguda vs. el acceso femoral en aquellos pacientes con SCA que precisan intervención invasiva ${ }^{34}$.

Resultados similares se encontraron en un meta-análisis que comparó el impacto del acceso radial versus el femoral sobre la incidencia de enfermedad renal aguda, demostrando que el uso del acceso radial redujo significativamente las tasas de enfermedad renal aguda (OR 0,51 IC 95\%, 0,39$0,67, p<0,0001)$, siendo más importante en los pacientes con SCAST (OR 0,42 IC 95\%, 0,24-0,72, $p=0,001$ ). Se concluyó que la ICP vía radial está asociada a menor incidencia de enfermedad renal aguda, ante todo en los pacientes con $\mathrm{SCAST}^{35}$.

\section{Conclusiones}

Los distintos estudios han demostrado las ventajas del acceso radial sobre el femoral en términos de reducción de morbilidad, mortalidad y complicaciones vasculares y hemorrágicas, con base en la importancia de la curva de aprendizaje para la obtención de estos resultados.

A pesar de esta evidencia científica, todavía no se cuenta con grandes estudios que enfaticen en la pérdida de la fuerza muscular y los estados disfuncionales de la extremidad superior intervenida; esto puede ser de gran importancia en el momento de escoger qué pacientes no se beneficiarían de esta técnica por las complicaciones funcionales que les pueda generar.

Se pone de manifiesto la necesidad de llevar a cabo estudios que traten de evaluar el verdadero compromiso en la funcionalidad, mejorando su detección temprana para enviar de forma oportuna a los servicios de rehabilitación, al igual que buscar dispositivos e introductores para el uso de accesos vasculares, que generen menos morbilidad, haciendo del acceso radial una vía mucho más segura.

\section{Conflicto de intereses}

Los autores declaran no tener ningún conflicto de intereses.

\section{Bibliografía}

1. Analisis de Situacion de Salud (ASIS) Colombia, 2015. Bogotá, noviembre de 2015 [Internet] [Acceso $17 \mathrm{Abr}$ 2017]. Disponible en: https://Www.minsalud.gov.co/sites/ rid/Lists/BibliotecaDigital/RIDE/VS/ED/PSP/asis-2015.pdf

2. Steg G, James SK, Atar D, Badano LP, Blomstrom Lundqvist C, Borger MA, et al. Guía de práctica clínica de la ESC para el 
manejo del infarto agudo de miocardio en pacientes con elevación del segmento ST. Rev Esp Cardiol. 2013;66:53.

3. Worner F, San Román A, Sánchez PL, Viana Tejedor A, GonzálezJuanatey JR. Atención a los pacientes con enfermedades cardiacas agudas y críticas. Posición de la Sociedad Española de Cardiología. Rev Esp Cardiol. 2016;69:239-42.

4. Vascular Access in Cardiac Catheterization and Intervention: Background, Indications, Contraindications. 10 de diciembre de 2016 [Acceso 17 Abr 2017]; Disponible en: http://emedicine. medscape.com/article/1894124-overview

5. Nathan S, Rao SV. Radial versus femoral access for percutaneous coronary intervention: Implications for vascular complications and bleeding. Curr Cardiol Rep. 2012;14:502-9.

6. Rondán J, Lozano I, Morís C, Martín M, Avanzas P, Suárez E. Cardiac catheterization via the right radial artery with a Judkins left catheter. A prospective study. Rev Esp Cardiol. 2005;58:868-71.

7. dos Santos MA, de Borba RP, de Moraes CV, Voltolini I, Azevedo EM, Cardoso CR, et al. Evaluation of radial artery patency after transradial catheterization. Rev Bras Cardiol Invasiva. 2012;20:403-7.

8. Jolly SS, Yusuf S, Cairns J, Niemelä K, Xavier D, Widimsky P, et al. Radial versus femoral access for coronary angiography and intervention in patients with acute coronary syndromes (RIVAL): A randomised, parallel group, multicentre trial. Lancet Lond Engl. 2011;377:1409-20.

9. Cardiac Interventions Today - Femoral Access Is Still a Contender [Internet]. Cardiac Interventions Today. [Acceso 23 May 2017]. Disponible en: http://citoday.com/2015/06/ femoral-access-is-still-a-contender/

10. Valgimigli M, Gagnor A, Calabró P, Frigoli E, Leonardi S, Zaro T, et al. Radial versus femoral access in patients with acute coronary syndromes undergoing invasive management: a randomised multicentre trial. The Lancet. 2015;385:2465-76.

11. Romagnoli E, Biondi-Zoccai G, Sciahbasi A, Politi L, Rigattieri $S$, Pendenza G, et al. Radial versus femoral randomized investigation in ST-segment elevation acute coronary syndrome: The RIFLE-STEACS (Radial Versus Femoral Randomized Investigation in ST-Elevation Acute Coronary Syndrome) study. J Am Coll Cardiol. 2012;60:2481-9.

12. Lee MS, Wolfe M, Stone GW. Transradial versus transfemoral percutaneous coronary intervention in acute coronary syndromes: Re-evaluation of the current body of evidence. JACC Cardiovasc Interv. 2013;6:1149-52.

13. Bleeding and Vascular Complications at the Femoral Access Site Following Percutaneous Coronary Intervention $(\mathrm{PCl})$ : An Evaluation of Hemostasis Strategies [Internet]. [Acceso 18 Abr 2017]. Disponible en: https://www.fda.gov/ downloads/ScienceResearch/SpecialTopics/UCM308944

14. May MRL, Singh K, Wells GA. Efficacy of Radial Versus Femoral Access in the Acute Coronary Syndrome. JACC Cardiovasc Interv. 2015;8:1405-9.

15. BrJCardiol. UK experience of conversion of radial to femoral access in coronary interventions [Internet]. The British Journal of Cardiology. [Acceso 25 May 2017]. Disponible en: https: / / bjcardio.co.uk/2015/08/uk-experience-of-conversion-of-radial -to-femoral-access-in-coronary-interventions/

16. Cardiac Interventions Today - Transradial Artery Catheterization in the United States [Internet]. Cardiac Interventions Today. [Acceso 23 May 2017]. Disponible en: http: / / citoday.com/2014/ 06/transradial-artery-catheterization-in-the-united-states/

17. 2013 ACCF/AHA Guideline for the Management of ST-Elevation Myocardial Infarction | JACC: Journal of the American College of Cardiology [Internet]. [Acceso 21 Abr 2017]. Disponible en: http://www.onlinejacc.org/content/61/4/e78

18. Guía ESC 2015 sobre el tratamiento de los síndromes coronarios agudos en pacientes sin elevación persistente del segmento ST. Rev Esp Cardiol. 2015; 68(12):1125-1125.
19. Bavishi C, Panwar SR, Dangas GD, Barman N, Hasan CM, Baber $U$, et al. Meta-Analysis of Radial Versus Femoral Access for Percutaneous Coronary Interventions in Non-ST-Segment Elevation Acute Coronary Syndrome. Am J Cardiol. 2016;117: 172-8.

20. Ferrante G, Rao SV, Jüni P, Da Costa BR, Reimers B, Condorelli G, et al. Radial Versus Femoral Access for Coronary Interventions Across the Entire Spectrum of Patients With Coronary Artery Disease: A Meta-Analysis of Randomized Trials. JACC CardiovasC Interv. 2016;9:1419-34.

21. Barbetta I, van den Berg JC. Access and Hemostasis: Femoral and Popliteal Approaches and Closure DevicesWhy, What, When, and How? Semin Interv Radiol. 2014;31: 353-60.

22. Claudia Jaramillo RG, Fernan Mendoza. Factores asociados a la presencia de sangrado clasificados por el Bleeding Academic Research Consortium, en pacientes con síndrome coronario agudo sometidos a intervención coronaria percutánea [Internet]. [Acceso $17 \mathrm{Abr}$ 2017]. Disponible en: http://www. sciencedirect.com/science/article/pii/S0120563316300390

23. Examining the Appropriateness of Radial or Femoral Access [Internet]. Medscape. [Acceso 18 Abr 2017]. Disponible en: http://www. medscape.com/viewarticle/778963

24. BrJCardiol. Risk factors for femoral arterial complications and management [Internet]. The British Journal of Cardiology. [Acceso 18 Abr 2017]. Disponible en: https://bjcardio.co.uk/ 2016/11/risk-factors-for-femoral-arterial-complications-andmanagement/

25. Yeni H, Axel M, Örnek A, Butz T, Maagh P, Plehn G. Clinical and Subclinical Femoral Vascular Complications after Deployment of two Different Vascular Closure Devices or Manual Compression in the Setting of Coronary Intervention. Int J Med Sci. 2016;13:255-9.

26. Rashid M, Kwok CS, Pancholy S, Chugh S, Kedev SA, Bernat I, et al. Radial Artery Occlusion After Transradial Interventions: A Systematic Review and Meta-Analysis. J Am Heart Assoc Cardiovasc Cerebrovasc Dis [Internet]. 25 de enero de 2016 [Acceso 30 May 2017];5(1). Disponible en: http://www.ncbi. $\mathrm{nlm}$.nih.gov/pmc/articles/PMC4859386/

27. Habib J, Baetz L, Satiani B. Assessment of collateral circulation to the hand prior to radial artery harvest. Vasc Med Lond Engl. 2012;17:352-61.

28. Dahm JB, Vogelgesang D, Hummel A, Staudt A, Völzke H, Felix SB. A randomized trial of 5 vs. 6 French transradial percutaneous coronary interventions. Catheter Cardiovasc Interv. 2002;57:172-6.

29. van Leeuwen MAH, van Mieghem NM, Lenzen MJ, Selles RW, Hoefkens MF, Zijlstra F, et al. The effect of transradial coronary catheterization on upper limb function. JACC Cardiovasc Interv. 2015;8:515-23.

30. Zwaan EM, IJsselmuiden AJJ, van Rosmalen J, van Geuns RJM, Amoroso G, Moerman E, et al. Rationale and design of the ARCUS: Effects of trAnsRadial perCUtaneouS coronary intervention on upper extremity function. Catheter Cardiovasc Interv Off J Soc Card Angiogr Interv. 2016;88:1036-43.

31. Zwaan EM, Koopman AGMM, Holtzer CAJ, Zijlstra F, Ritt MJPF, Amoroso G, et al. Revealing the impact of local access-site complications and upper extremity dysfunction post transradial percutaneous coronary procedures. Neth Heart J Mon J Neth Soc Cardiol Neth Heart Found. 2015;23:514-24.

32. Sirker A, Kwok CS, Kotronias R, Bagur R, Bertrand O, Butler R, et al. Influence of access site choice for cardiac catheterization on risk of adverse neurological events: A systematic review and meta-analysis. Am Heart J. 2016;181:107-19.

33. Upper extremity dysfunction from transradial $\mathrm{PCl}$ underappreciated [Internet]. [Acceso 15 May 2017]. Disponible en: http: // www.mdedge.com/ecardiologynews/article/109135/ interventional-cardiology-surgery/upper-extremity-dysfunction 
34. Andò G, Cortese B, Russo F, Rothenbühler M, Frigoli E, Gargiulo $G$, et al. Acute Kidney Injury After Radial or Femoral Access for Invasive Acute Coronary Syndrome Management: AKI-MATRIX. J Am Coll Cardiol. 2017.
35. Andò G, Costa F, Trio O, Oreto G, Valgimigli M. Impact of vascular access on acute kidney injury after percutaneous coronary intervention. Cardiovasc Revascularization Med Mol Interv. 2016;17:333-8. 